\title{
Síntomas depresivos y funcionamiento cognitivo de las personas en proceso de reintegración mayores (PPRM) de Colombia ${ }^{i}$ Depressive Symptoms and Cognitive Functioning of Senior Citizens in the Reintegration Process (PPRM) in Colombia.
}

\section{Por: Carlos Robledo Marín, ${ }^{1}$ Doris Cardona Arango, ${ }^{2}$ Alejandra Segura Cardona ${ }^{3}$ \& Douglas Lizcano Cardona ${ }^{4}$}

1. Licenciado en Educación, Especialista en Gerencia de la Protección Social, Magister en Desarrollo. Doctor en Humanidades. Director Ejecutivo Fundacol, Medellín, Colombia ORCID: http://orcid.org/0000-0002-6944-561X Scholar: https://scholar.google.com/citations?user=606yPhEAAAA]\&hl=es Contacto: direccionejecutiva@ fundacol.com

2. Administradora de Empresas, Magíster en Epidemiología, Magíster en Salud Pública, Doctora en Demografía. Docente Universidad CES, Medellín, Colombia ORCID https://orcid.org/0000-0003-4338-588X Scholar: https://scholar.google.es/citations?user=72K9kikAAAA]\&hl=es\&oi=sra Contacto: dcardona@ces.edu.co

3. Psicóloga, Magíster en Epidemiología. Docente Universidad CES. Medellín, Colombia ORCID: https://orcid.org/0000-0002-1624-0952 Scholar: https://scholar.google.es/ citations? user=AB_byLOAAAA]\&hl=es\&oi=sra Contacto: dsegura@ces.edu.co

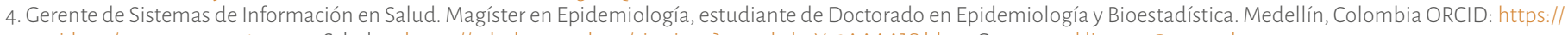
orcid.org/0000-0002-1652-3231 Scholar: https://scholar.google.es/citations?user=k7h4Y_8AAAA]\&hl=es Contacto: d.lizcano@uces.edu.co.

\section{OPEN ACCESS}

\section{(c) $(1) \Theta \Theta$}

Copyright: (C) 2020 El Ágora USB.

La Revista El Ágora USB proporciona acceso abierto a todos sus contenidos bajo los términos de la licencia creative commons Atribución-NoComercial-SinDerivar 4.o Internacional (CC BY-NC-ND 4.0)

Tipo de artículo: Investigación

Recibido: marzo de 2020

Revisado: abril de 2020

Aceptado: junio de 2020

Doi: $10.21500 / 16578031.5134$

Citar así: Robledo Marín, C., Cardona Arango,

D. Segura Cardona, A. \& Lizcano Cardona, D.

(2020). Síntomas depresivos y funcionamiento cognitivo de las personas en proceso de reintegración mayores (PPRM) de Colombia. El Ágora USB, 20(2). 118-128

Doi: $10.21500 / 16578031.5134$

\section{Resumen}

Algunas personas en procesos de reintegración mayores (PPRM) que participaron en la guerra presentan alteraciones mentales explicadas por el largo tiempo desescolarizados y las situaciones vividas, que le disminuyen la posibilidad de acceso al campo laboral y a la sociedad, el funcionamiento cognitivo alterado fue más prevalente en personas sin educación formal, sin pareja o vivir en familias no funcionales. Entre los factores demográficos de las PPRM que aumentan la probabilidad de tener un síntoma depresivo fue pertenecer al sexo masculino, tener más de 65 años, no contar con apoyo de una pareja y disminuye esta probabilidad si se cuenta con algún grado de escolaridad. De los factores familiares y sociales que se analizaron, solo se encontró que vivir con una familia no funcional aumenta el riesgo de sufrir síntomas depresivos. Por lo que se requiere acompañamiento para la aceptación de su proceso de envejecimiento, el aprovechamiento de experiencias y el ejercicio de la ciudadanía. Palabras clave: Envejecimiento; reintegración; síntomas depresivos; funcionamiento cognitivo; guerra.

\section{Abstract}

Some Senior Citizens in Reintegration Processes (PPRM), who participated in war have mental disturbances explained by the long de-schooling and living situations, which decrease the possibility of accessing the workforce and society. Altered cognitive functioning was more prevalent in people without formal education, without a partner, or living in non-functional families. Among the PPRM demographic factors of PPRM, which increase the likelihood of having a depressive symptom was belonging to the male sex, being over 65 years old, not having support from a partner. This likelihood decreases if there is any degree of schooling. Among the family and social factors discussed, only living with a non-functional family was found to increase the risk of depressive symptoms. Thus, accompaniment is required for the acceptance of their aging process, the use of experiences, and the exercise of citizenship.

Keywords: Aging; Reintegration; Depressive Symptoms; Cognitive Functioning; and War. 


\section{Introducción}

Los efectos del conflicto armado sobre las personas que lo viven, pueden ser irreversibles (Somasundaram y Sivayo kan, 2013; Wilmer, 1998), dada la exposición a factores de riesgo psicosocial (World Health Organization. 2004), pudiéndose presentar alteraciones del comportamiento, estrés, depresión, demencia, agresividad o alteraciones del sueño, entre otras, haciéndose necesarios los cuidados permanentes de las personas mayores por parte de profesionales calificados, de instituciones geriátricas especializadas, o mediante el fortalecimiento de las redes de apoyo familiar y comunitario (Deeny y McFetridge, 2008; Nakayama et al., 2014; Wilmer, 1998).

De acuerdo con investigaciones de otros países, para los excombatientes en general, las afecciones en su salud mental pueden ser: estrés postraumático, ansiedad, trastornos del sueño (insomnio, hiperinsomnio, pesadillas), depresión, y los trastornos del estado del ánimo y la personalidad, entre otros (Patel et al., 2007; Senarath, Wickramage y Peiris, 2014). También se evidencia consumo de alcohol y sustancias psicoactivas, con las que se profundizan las conductas antisociales, la dificultad para controlar impulsos, y los sentimientos de culpa que afectan la autoestima y la readaptación emocional y social de las personas que han participado directamente en los conflictos armados (veteranos o excombatientes) (Korineky Teerawichitchainan, 2014; Preston, 1997). En el caso específico de las personas adultos mayores colombianas, el Programa de Reintegración reconoce una especial protección y acompañamiento, a través de la Resolución 1356 de 2016 (Agencia Colombiana para la Reintegración, 2016), modificatoria de la Resolución 0754 de 2013 (Agencia Colombiana para la Reintegración, 2013), inciso segundo del artículo 4, el cual resalta el beneficio de acompañamiento psicosocial con componente específico, desarrollados a través de un plan de trabajo con énfasis en salud y bienestar integral.

La salud mental en la etapa de la vejez, es tan importante como en las otras etapas de la vida; existen factores sociales, psíquicos y biológicos que determinan la salud mental en las personas mayores y las deficiencias en este aspecto pueden tener repercusiones a nivel físico, psicológico y social; esta etapa en muchas ocasiones está acompañada por la pérdida de independencia, dolor a causa de diferentes enfermedades, fragilidad, discapacidad y/o necesidad de asistencia continua; situaciones que ocasionan angustia y aislamiento.

Los problemas de salud mental en las personas mayores se han pasado por alto, siendo interpretados como algo propio de la edad; en esta etapa los problemas mentales y físicos suele entrelazarse, hasta el punto de llegar a ser difícil la separación de un problema psicológico a una enfermedad física (Belsky, 1990). La salud mental puede ser mejorada por medio de hábitos saludables y entornos de vida que promuevan el bienestar (Organización Mundial de la Salud, 2017a). Según el Departamento de Salud Mental y Abuso de Sustancias de la Organización Mundial de la Salud (OMS), la exposición a situaciones estresantes como lo padecen las personas expuestas a la guerra, es un factor de riesgo para desarrollar tanto problemas sociales como de salud mental (Organización Mundial de la Salud, 2017b).

Las consecuencias psicosociales de la guerra en los actores del conflicto armado se han asumido de manera silenciosa, es decir, no se habla de ellos, por factores como el miedo o la indiferencia social, pasando desapercibida la forma cómo se ve afectada sustancialmente la cotidianidad y por ende la salud mental, de los sujetos que hacen o hicieron parte del conflicto armado. Es importante anotar que al momento de la desmovilización, se presentan estigmas sociales que dificultan la adaptación de algunas personas (Aristizábal, Howe y Palacio, 2009); por esta razón, este estudio busca caracterizar los síntomas depresivos y funcionamiento cognitivo alterado de las PPRM, que afectan su salud mental, asociadas a características demográficas y sociales. 


\section{Metodología}

Se realizó un estudio cuantitativo, analítico, transversal, con fuente de información primaria, correspondiente a 252 personas encuestadas en cinco CT de Colombia: Antioquia-Chocó, CesarCuajira, Cundinamarca-Boyacá, Meta-Orinoquía y Valle del Cauca, a las cuales se les indagó por variables demográficas y sociales, y variables de salud mental como: funcionamiento cognitivo (Escala MMSE modificado), nivel de depresión (Escala de Depresión Ceriátrica Abreviada) y también se aplicó escala de medición del funcionamiento familiar (APGAR familiar). La encuesta fue aplicada por encuestadores estandarizados y también se realizó prueba piloto.

El procesamiento de la información fue realizado en el software SPSS versión 21.0. Se realizó análisis de frecuencias absolutas y relativas, se tomó como variable dependiente el resultado de las escalas aplicadas y posteriormente se realizó el análisis bivariado, apoyada en la prueba estadística chi cuadrado $\left(X^{2}\right)$ y para identificar las asociaciones de esta variable con las características demográficas y sociales, se consideró un valor de p menor de 0,05 como estadísticamente significativo. Así mismo se calculó la medida epidemiológica (razón de prevalencia), con el fin de mirar los factores de aumentan la probabilidad de padecer un trastorno mental.

\section{Resultados}

\section{Características demográficas y sociales}

Los hombres representaron el 84,5\% (213) y las mujeres el 15,5\% (39), con una relación de masculinidad de 5,5 hombres por cada mujer. Por estado civil, el 43,3\% convivían en unión libre seguidos de los solteros y de aquellos que manifestaron estar separados o divorciados. El promedio de edad fue de 57,63 años (DE 6,9 años); los hombres presentaron 57,95 años y las mujeres 55,92 años. Tabla 1.

Con respecto al máximo nivel educativo alcanzado, la mitad de los PPRM han cursado la secundaria (50,4\%) y el 39,7\% la primaria; el nivel educativo que tuvo una menor frecuencia en la muestra fue el universitario o superior y solo un 7,1\% manifestó no tener ningún nivel de educación formal. Las mujeres presentaron un porcentaje mayor de formación secundaria que los hombres y en ambos sexos, el orden en los niveles de formación es similar. Tabla 1.

El 90,1\% (227) manifestó que sabía leer y escribir; el 86,5\% (218) consideró que pueden realizar cálculos matemáticos básicos y el 69,9\% (176) comprende el significado de las lecturas que realiza y solo el 42,9\% (108) de los encuestados manifestó que le gustaría continuar sus estudios. Ver Tabla 1.

Al analizar el nivel educativo por CT, se encontró que en el Valle del Cauca se encuentra la proporción más alta de PPRM sin ningún nivel de escolaridad (16,7\%); Meta-Orinoquía es el CT con el porcentaje más alto de personas con nivel de primaria (50\%); en Cesar-Cuajira y Antioquia-Chocó el mayor porcentaje tenía formación secundaria, con un $56 \%$ y $56,8 \%$ respectivamente y la formación universitaria fue porcentualmente mayor en Cundinamarca-Boyacá (7\%). Tabla 1.

\section{Tabla 1.}

Distribución porcentual de las personas en proceso de reintegración mayores, según características demográficas y sociales por sexo. Colombia.

\begin{tabular}{lllllll}
\hline & \multicolumn{2}{l}{ Sexo } & & \multirow{2}{*}{ Total } \\
\cline { 2 - 5 } Características demográficas y sociales & \multicolumn{2}{l}{ Hombre } & \multicolumn{2}{c}{ Mujer } & & \\
\cline { 2 - 5 } & $\mathbf{N}$ & $\%$ & $\mathbf{N}$ & $\%$ & $\mathbf{N}$ & $\%$ \\
\hline Estado civil & 56 & 26,3 & 11 & 28,2 & 67 & 26,6 \\
Soltero/a & 27 & 12,7 & 6 & 12,8 & 32 & 12,7
\end{tabular}




\begin{tabular}{|c|c|c|c|c|c|c|}
\hline Unión libre & 97 & 45,5 & 12 & 30,8 & 109 & 43,3 \\
\hline Separado/a-Divorciado & 28 & 13,1 & 7 & 17,9 & 35 & 13,9 \\
\hline Viudo/a & 5 & 2,3 & 4 & 10,3 & 9 & 3,6 \\
\hline Total & 213 & 100,0 & 39 & 100,0 & 252 & 100,0 \\
\hline \multicolumn{7}{|c|}{ Máximo nivel educativo } \\
\hline Ninguna & 16 & 7,5 & 2 & 5,1 & 18 & 7,1 \\
\hline Primaria & 89 & 41,8 & 11 & 28,2 & 100 & 39,7 \\
\hline Secundaria & 102 & 47,9 & 25 & 64,1 & 127 & 50,4 \\
\hline Terciaria & 6 & 2,8 & 1 & 2,6 & 7 & 2,8 \\
\hline Total & 213 & 100,0 & 39 & 100,0 & 252 & 100,0 \\
\hline
\end{tabular}

Fuente: elaboración propia.

\section{Funcionamiento cognitivo}

El promedio de años de educación formal se ha relacionado con el funcionamiento cognitivo alterado, por ello vale recordar que durante varios años, las PPRM estuvieron desescolarizados con poco número de años aprobados que pudieran explicar las alteraciones cognitivas encontradas; en los hombres, el promedio fue de 4,55 años (DE 2,3 años) y en las mujeres, el promedio de años fue de 5,26 años (DE 2,6 años). Tabla 2.

Según funcionamiento cognitivo de las PPRM, medido con la escala MMSE, se encontró que el 40,1\% (101) presentan alteraciones, mientras que el 59,9\% (151) no lo tienen. Por sexo, las mujeres presentaron una prevalencia de $48,7 \%$ y en los hombres fue de $38,5 \%$ sin diferencias estadísticas. Así mismo, los menores de 65 años también tienen menor riesgo, en los que no tienen educación se aumenta la probabilidad del riesgo en un $11,8 \%$ con respecto a los que presentaron algún nivel educativo y no tener pareja también aumenta dicha probabilidad de tener riesgo de funcionamiento cognitivo alterado. Ninguna relación es estadísticamente significativa. Tabla 2.

Al ajustar por las demás variables, no se percibe mayor variación en el funcionamiento cognitivo en la población estudiada, pero es posible que se aumenten las alteraciones en las personas sin educación y en quienes se encuentra solas (sin pareja); además el ser menor de 65 años se considera un aspecto que reduce la alteración, lo cual coincide con lo reportado en la literatura. Ver Tabla 2.

\section{Tabla 2.}

Distribución proporcional de las personas en proceso de reintegración mayores, según aspectos demográficos por riesgo de funcionamiento cognitivo alterado. Colombia

\begin{tabular}{|c|c|c|c|c|c|c|c|c|c|c|}
\hline \multicolumn{2}{|c|}{ Aspectos demográficos } & \multicolumn{4}{|c|}{$\begin{array}{c}\text { Funcionamiento cognitivo } \\
\text { alterado }\end{array}$} & \multirow{3}{*}{$\begin{array}{c}\mathrm{X}^{2} \\
\text { (valor } \mathrm{p})\end{array}$} & \multirow{3}{*}{ RP crudo } & \multirow{3}{*}{ IC 95\% } & \multirow{3}{*}{$\begin{array}{l}\text { RP ajusta- } \\
\text { do }\end{array}$} & \multirow{3}{*}{ IC 95\% } \\
\hline & & \multicolumn{3}{|c|}{ Si } & \multirow{2}{*}{$\begin{array}{l}\text { No } \\
\% \\
\end{array}$} & & & & & \\
\hline & & $\mathbf{n}$ & $\%$ & $\mathbf{n}$ & & & & & & \\
\hline \multirow{2}{*}{ Sexo } & Hombre & 82 & 38,5 & 131 & 61,5 & \multirow{2}{*}{$\begin{array}{l}1,434 \\
(0,231)\end{array}$} & 0,790 & $0,549-1,137$ & 0,659 & $0,328-1,326$ \\
\hline & Mujer & 19 & 48,7 & 20 & 51,3 & & 1,000 & - & 1,000 & - \\
\hline \multirow[t]{2}{*}{ Edad } & $<65$ años & 80 & 37,6 & 133 & 62,4 & \multirow[t]{2}{*}{$\begin{array}{l}3,641 \\
(0,056)\end{array}$} & 0,698 & $\begin{array}{l}0,497- \\
0,978\end{array}$ & 0,522 & $\begin{array}{l}0,260- \\
1,049\end{array}$ \\
\hline & $>65$ años & 21 & 53,8 & 18 & 46,2 & & 1,000 & - & 1,000 & - \\
\hline \multirow{2}{*}{ Educación } & Sin educación & 8 & 44,4 & 10 & 55,6 & \multirow{2}{*}{$\begin{array}{l}0,154 \\
(0,695)\end{array}$} & 1,118 & $0,652-1,919$ & 1,156 & $0,431-3,099$ \\
\hline & Con educación & 93 & 39,7 & 141 & 60,3 & & 1,000 & - & 1,000 & - \\
\hline \multirow{2}{*}{ Estado civil } & Sin pareja & 51 & 45,9 & 60 & 54,1 & \multirow{2}{*}{$\begin{array}{l}2,843 \\
(0,092)\end{array}$} & 1,296 & $\begin{array}{l}0,959- \\
1,750\end{array}$ & 1,444 & $0,861-2,422$ \\
\hline & Con pareja & 50 & 35,5 & 91 & 64,5 & & 1,000 & -- & 1,000 & -- \\
\hline
\end{tabular}

Fuente: elaboración propia. 
Se relacionó el funcionamiento cognitivo alterado y algunos aspectos familiares y sociales, encontrando mayor probabilidad en PPRM que viven con familias menos funcionales $(48,5 \%)$ en comparación con aquellos de familias funcionales (29,0\%), según la escala APGAR familiar, siendo significativa la diferencia $\left(X^{2}=9,389 p=0,002\right)$, con una relación de 2,3 personas de familia menos funcionales con funcionamiento cognitivo alterado, por cada persona de familia funcional con funcionamiento cognitivo alterado ( $\mathrm{RP}=2,306 \mathrm{IC} 95 \% 1,345-3,953)$. Tabla 3.

En cuanto al número de amigos cercanos, se identificó mayor porcentaje de deterioro en quienes manifestaron tener más de tres amigos cercanos $(41,0 \%)$ que en aquellos que dijeron tener tres o menos $(37,7 \%)$ sin ser significativa la diferencia $\left(X^{2}=0,228 p=0,633\right)$. Quienes manifestaron tener cerca tres o menos familiares cercanos, tuvieron un porcentaje de funcionamiento cognitivo alterado de $42,3 \%$, superior al de aquellos que tenían más de tres familiares (39,2\%), sin ser significativa la diferencia $\left(X^{2}=0,195 p=0,659\right)$. El mayor porcentaje de personas con deterioro se encontró en quienes vivían solos (45,1\%) seguido de quienes vivían con familias extensas (37,3\%), de las familias monoparentales el 45,1\% tenían funcionamiento cognitivo alterado, la familia reconstruida fue un 35,7\% y los de familia nuclear mostraron el porcentaje más bajo de deterioro con un 31,4\%, sin embargo, las diferencias no fueron significativas $\left(X^{2}=2,607 p=0,626\right)$. Tabla 3 .

Tabla 3.

Funcionamiento cognitivo alterado en personas en proceso de reintegración mayores, según aspectos familiares y sociales. Colombia

\begin{tabular}{|c|c|c|c|c|c|c|c|c|c|}
\hline \multicolumn{2}{|c|}{ Aspectos familiares y sociales } & \multicolumn{4}{|c|}{$\begin{array}{l}\text { Funcionamiento cognitivo } \\
\text { alterado }\end{array}$} & \multirow[t]{3}{*}{$\mathbf{X}^{2}$} & \multirow[t]{3}{*}{ Valor $\mathbf{p}$} & \multirow[t]{3}{*}{ RP crudo } & \multirow[t]{3}{*}{ IC 95\% } \\
\hline & & \multicolumn{2}{|l|}{ Si } & \multicolumn{2}{|r|}{ No } & & & & \\
\hline & & $\mathbf{n}$ & $\%$ & $\mathbf{n}$ & $\%$ & & & & \\
\hline $\begin{array}{l}\text { Funcionamiento } \\
\text { familiar }\end{array}$ & Funcional & 40 & 29,0 & 98 & 71,0 & 9,389 & $0,002^{*}$ & 1,000 & - \\
\hline \multirow{2}{*}{ Amigos cercanos } & Tres o menos & 26 & 37,7 & 43 & 62,3 & \multirow{2}{*}{0,228} & \multirow{2}{*}{0,633} & 1,000 & - \\
\hline & Más de tres & 75 & 41,0 & 108 & 59,0 & & & 1,149 & $0,650-2,029$ \\
\hline \multirow{2}{*}{ Familiares cercanos } & Más de tres & 71 & 39,2 & 110 & 60,8 & \multirow{2}{*}{0,195} & \multirow{2}{*}{0,659} & 1,000 & - \\
\hline & Tres o menos & 30 & 42,3 & 41 & 57,7 & & & 1,134 & $0,649-1,980$ \\
\hline \multirow[t]{3}{*}{ Tipo de familia } & Reconstruida & 5 & 35,7 & 9 & 64,3 & \multirow[t]{3}{*}{2,607} & 0,748 & 1,214 & $0,371-3,968$ \\
\hline & Monoparental & 7 & 36,8 & 12 & 63,2 & & 0,647 & 1,275 & $0,452-3,597$ \\
\hline & Unipersonal & 23 & 45,1 & 28 & 54,9 & & 0,109 & 1,795 & $0,878-3,670$ \\
\hline
\end{tabular}

*Valor $p<0,05$

Fuente: elaboración propia.

\subsection{Síntomas depresivos}

Se encontró que el nivel de depresión evaluado con la escala de depresión geriátrica abreviada, evidencia síntomas depresivos en las PPRM en un 73,6\% (176) presentando riesgo de alteración en su estado de ánimo del tipo depresivo, mientras que el 26,4\% (63) no lo presentan. No se evidenció asociación estadística del riesgo de depresión según variables demográficas, pero si es llamativo que las PPRM que no tienen educación tienen mayor probabilidad de presentar el riesgo (11,2\%). Tabla 4.

Al analizar el riesgo de síntomas depresivos en los PPRM y luego de ajustar por las demás variables estudiadas, se encontró que según sexo se presenta más riesgo de presentar alteración anímica 
en los hombres; en cuanto a la edad el mayor riesgo se presenta en los mayores de 65 años, con menores niveles de escolaridad y con menor apoyo social por parte de una pareja. Tabla 4.

Al analizar las PPRM de acuerdo a los síntomas depresivos o riesgo de depresión observado, se encontró que fue mayor el porcentaje de personas con depresión en aquellas de familias menos funcionales $(82,8 \%)$ en comparación con aquellos de familias funcionales $(67,4 \%)$ siendo significativa la diferencia $\left(X^{2}=7,111 p=0,008\right)$; se observó que por cada persona con síntomas perteneciente a una familia funcional hay 2,3 personas que vive con familias menos funcionales ( $R P=2,334$; IC $95 \%$ 1,241-4,391). Tabla 4.

Tabla 4.

Distribución proporcional de las personas en proceso de reintegración mayores, según aspectos demográficos por síntomas depresivos. Colombia, cinco grupos territoriales

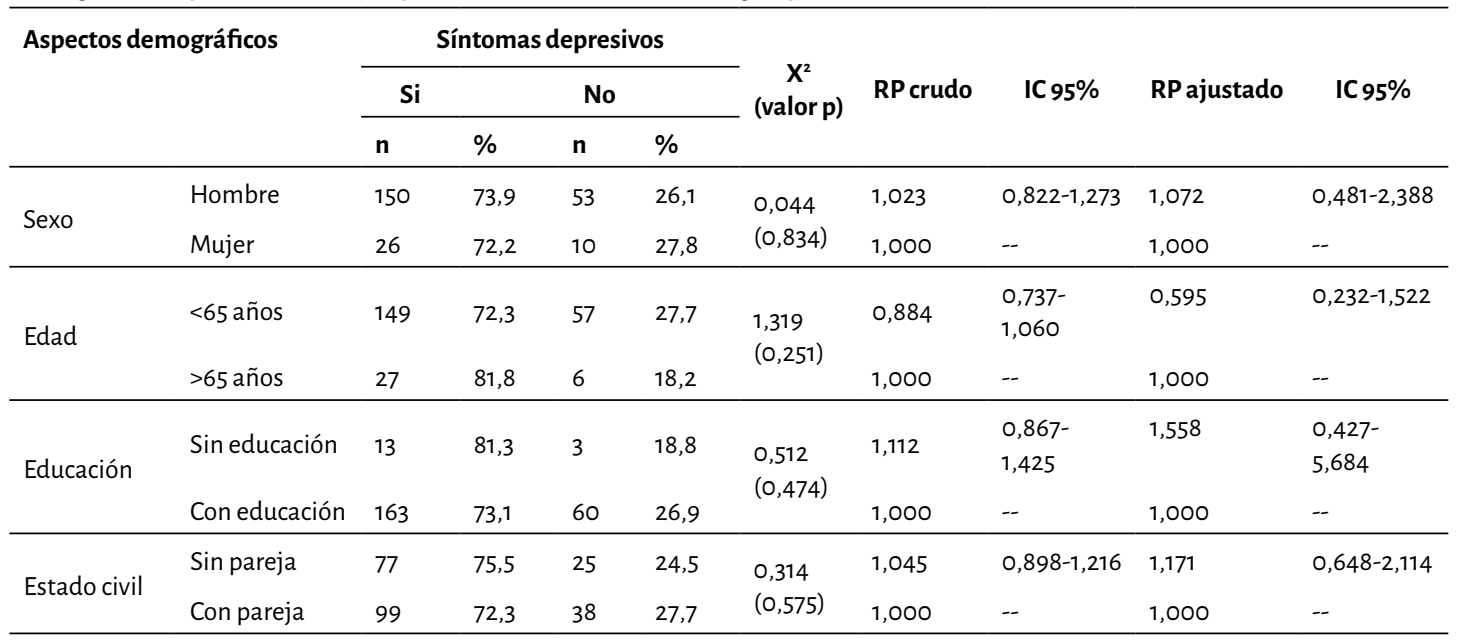

Fuente: elaboración propia.

En cuanto al número de familiares cercanos, aunque se observó mayor porcentaje de depresión en aquellos que manifestaron tener tres o menos amigos cerca $(75,4 \%)$, la diferencia con los que manifestaron tener más de tres amigos y mostraron depresión (72,9\%) no fue estadísticamente significativa $\left(X^{2}=0,148 p=0,700\right)$. De aquellos con tres o menos familiares cercanos, el $81,7 \%$ mostraron riesgo de depresión, mientras que en quienes tenían más de tres familiares cercanos el porcentaje de depresión fue del 70,2\%, sin embargo, la diferencia no fue significativa $\left(X^{2}=3,372 p=0,066\right)$. Tabla 5 .

De acuerdo al tipo de familia, se observó mayor porcentaje de personas con síntomas depresivos en quienes pertenecían a familias extensas $(80,6 \%)$, seguido de aquellos de familia unipersonal $(76,5 \%)$, familia nuclear $(70,9 \%)$, reconstruida $(64,3 \%)$ y el menor porcentaje encontrado fue en las familias monoparental (57,9\%). No se encontró asociación estadística entre síntomas y factores familiares y sociales. Tabla 5. 
Tabla 5.

Síntomas depresivos en personas en proceso de reintegración mayores, según aspectos familiares y sociales. Colombia

\begin{tabular}{|c|c|c|c|c|c|c|c|c|c|}
\hline \multirow[t]{3}{*}{$\begin{array}{c}\text { Aspectos familiares y } \\
\text { sociales }\end{array}$} & & \multicolumn{4}{|c|}{ Síntomas depresivos } & \multirow{3}{*}{$\mathbf{X}^{2}$} & \multirow{3}{*}{ Valor $p$} & \multirow{3}{*}{ RP crudo } & \multirow{3}{*}{ IC $95 \%$} \\
\hline & & \multicolumn{2}{|c|}{ Si } & \multicolumn{2}{|c|}{ No } & & & & \\
\hline & & $\mathbf{n}$ & $\%$ & $\mathbf{n}$ & $\%$ & & & & \\
\hline \multirow{2}{*}{$\begin{array}{l}\text { Funcionamiento } \\
\text { familiar }\end{array}$} & Funcional & 93 & 67,4 & 45 & 32,6 & \multirow{2}{*}{7,111} & \multirow{2}{*}{$0,008^{*}$} & 1,000 & - \\
\hline & Disfuncional & 82 & 82,8 & 17 & 17,2 & & & 2,334 & $1,241-4,391$ \\
\hline \multirow{2}{*}{ Amigos cercanos } & Más de tres & 124 & 72,9 & 46 & 27,1 & \multirow{2}{*}{0,148} & \multirow{2}{*}{0,700} & 1,000 & - \\
\hline & Tres o menos & 52 & 75,4 & 17 & 24,6 & & & 1,135 & $0,596-2,160$ \\
\hline \multirow{2}{*}{ Familiares cercanos } & Más de tres & 118 & 70,2 & 50 & 29,8 & \multirow{2}{*}{3,372} & \multirow{2}{*}{0,066} & 1,000 & -- \\
\hline & Tres o menos & 58 & 81,7 & 13 & 18,3 & & & 1,890 & $0,952-3,755$ \\
\hline \multirow{5}{*}{ Tipo de familia } & Nuclear & 61 & 70,9 & 25 & 29,1 & \multirow{5}{*}{5,230} & 0,265 & 1,000 & - \\
\hline & Extensa & 54 & 80,6 & 13 & 19,4 & & 0,172 & 1,702 & $0,793-3,654$ \\
\hline & Reconstruida & 9 & 64,3 & 5 & 35,7 & & 0,616 & 0,738 & $0,225-2,420$ \\
\hline & Monoparental & 11 & 57,9 & 8 & 42,1 & & 0,272 & 0,564 & $0,203-1,567$ \\
\hline & Unipersonal & 39 & 76,5 & 12 & 23,5 & & 0,481 & 1,332 & $0,600-2,956$ \\
\hline
\end{tabular}

*Valor $\mathrm{p}<0,05$

Fuente: elaboración propia.

\section{Discusión}

Las consecuencias de la guerra en los combatientes son tema de preocupación desde la primera guerra mundial; uno de las principales efectos es el deterioro mental de los actores, lo cual ha sido experimentado por los desmovilizados colombianos tras más de 55 años de conflicto irregular (Baldovino, 2014). La Organización Mundial de la Salud (OMS) calcula que en este tipo de conflictos, el 10\% de las personas que viven experiencias traumáticas suelen sufrir graves problemas de salud mental y otro 10\% comportamientos que dificultarán su capacidad para vivir con normalidad (Peña, Espíndola, Cardoso y González, 2007).

Por esta razón, se compararán los hallazgos de PPRM con resultados de estudios realizados en excombatientes, aclarando que si bien no se encontraron diferencias estadísticamente significativas, dadas principalmente por el tamaño de la muestra, si es posible mostrar diferencias y similitudes con ellos.

Los síntomas de alteraciones mentales en excombatientes son más evidentes en mujeres que en hombres (Amar, Llanos, Ávila-Toscano, Orozco, 2011; Pittman, Goldsmith, Lemmer, Kilmer, Baker, 2012), como en este estudio donde las mujeres presentaron una prevalencia del $48,7 \%$ de alteraciones cognitivas. Varias explicaciones han sido propuestas para justificar el efecto del sexo en el desarrollo de alteraciones mentales en excombatientes: las mujeres muestran tasas más altas de exposición a eventos traumáticos (Creamer, Burgess y McFarlane, 2001); mayor historial de trastornos psiquiátricos como ansiedad y depresión; y mayor tendencia a asumir respuestas emocionales de miedo, impotencia u horror ante un evento traumático (Baldovino, 2014).

Fontana, Rosenheck y Desai (2010) encontraron diferencias entre la presencia de psicopatologías en veteranos femeninos y masculinos de la guerra de Iraq y Afganistán, donde las mujeres tuvieron menos apoyo interpersonal y económico, fueron más expuestas a traumas y a diferentes niveles de patologías que los evaluados en hombres, aunque tuvieron menos síntomas de estrés postraumático, abuso y dependencia de alcohol y más frecuencia de trastornos del estado de ánimo como ansiedad y depresión. Otro estudio revela que la guerra de Nicaragua, conflicto de baja 
intensidad a nivel mundial, no dejó de ser aterrorizante para la población general durante 1980, donde el $62 \%$ de la población masculina y 91\% de la femenina presentó alteraciones afectivas y cognitivas (Summerfield y Toser, 1991).

Uno de los estudios más importantes realizado con veteranos de guerra de Vietnam, evaluó, a través de entrevistas a 3016 veteranos en cincuenta estados de Estados Unidos y Puerto Rico, encontrando que el 30,6\% de soldados varones y el 26,9\% de mujeres sufrieron los síntomas afectivos en algún momento luego del servicio militar. La mitad de los hombres (45\%) presentaron multimorbilidad mental con depresión mayor, estrés postraumático, abuso y dependencia de sustancias alcohólicas, desordenes de conducta y alteraciones cognitivas; abuso y dependencias de drogas (35\%), fobia simple (31\%), fobia social (28\%) y distimia (21\%) (Kulka et al., 1990).

Igualmente lo muestra un estudio realizado con 25 veteranos de la guerra Irán-Iraq, con miembros amputados, donde se encontró que el 35\% habían tenido una historia de depresión, estrés postraumático y alteración cognitiva (Ebrahimzadeh, Fattahi y Nejad, 2006). En Colombia uno de los trastornos mentales más frecuentes en excombatientes es el estrés postraumático (Jiménez, 2009).

Según varias asociaciones de veteranos de guerra y médicos militares y un estudio sobre la salud mental de las tropas elaborado por el Ejército de Tierra de EEUU, uno de cada seis soldados que han servido en Irak, muestra síntomas de depresión clínica grave; el documento indica que esta cifra puede aumentar a uno de cada tres (Peña et al.,2007).

El 35\% de los combatientes de Iraq recibieron atención de salud mental durante el primer año y el 12\% de los más de 220.000 participantes del estudio se les diagnosticó algún trastorno mental. El 19\% de los que regresaron de Iraq reportaron problemas de salud mental, comparado con el 11\% de los que volvieron de Afganistán y el 8,5\% de los que regresaron de Bosnia y otros lugares (Reeves, Parkery Konkle-Parker, 2016).

En lo cognitivo y como consecuencia de la guerra, se han manifestado varias alteraciones psiquiátricas; se han descrito estados de "obnubilación cognitiva" caracterizados por confusión, desorientación en el tiempo, dificultad para tomar decisiones y lentitud del pensamiento; y otra manifestación de "adormecimiento psíquico" caracterizado por un sentimiento de desinterés, pobre respuesta afectiva y sensación de distanciamiento de los demás (Stetz, McDonald, Lukey, Cifford, 2005). En este estudio fue más alta la prevalencia de funcionamiento cognitivo alterado en las mujeres $(48,7 \%)$ que en los hombres $(38,5 \%)$ superior a la reportada por el estudio Nacional de Salud, Bienestar y Envejecimiento (SABE), realizado en Colombia en el año 2015 donde el deterioro leve fue del 17\% en personas de 60 años y más de edad (Ministerio de Salud y Protección Social et al., 2016a).

A nivel nacional, un estudio realizado con una muestra de 109 adultos reclutados en la Unidad de Atención y Orientación (UAO) a la población desplazada en Medellín mostró que el sexo femenino era un predictor significativo de niveles más altos de síntomas afectivos y mentales, y que ser mujer, tener poca educación y los separados, en comparación con los casados, predijeron mayores niveles de síntomas emocionales y cognitivos (Richards, 2011).

Pensar en el momento nacional actual, los medios de comunicación intentan realizar llamados de alerta con el fin de fomentar la importancia de la intervención desde el punto de vista mental, con titulares como La salud mental en Colombia, un enorme desafío del posconflicto donde afirman que las víctimas o actores del conflicto armado, traducidos en millones de colombianos, sufren los traumas de la guerra que sólo ahora tendrán que empezar a cicatrizar (Semana, 2017).

\section{Conclusiones}

Dado el aporte demográfico de la población adulta mayor en el país, es necesaria una mayor capacitación y acompañamiento psicosocial a los PPRM en estrategias de afrontamiento para: aceptación del proceso de envejecimiento natural que viven, abordaje de su funcionamiento cognitivo y de síntomas depresivos, ruptura de las redes de apoyo social y familiar, pérdida de 
capacidades y habilidades para la incursión en el campo laboral, aprovechamiento de experiencias y conocimientos en áreas específicas, ejercicio de la ciudadanía y los derechos humanos.

Así mismo, un acompañamiento constante a las familias de las PPRM para la adherencia a programas y proyectos de la oferta pública a través de los reintegradores, donde se les enseñe: aceptación de los cambios físicos y mentales de los PPRM, cambio de roles familiares cuando se regresa al hogar después de un tiempo por fuera, vivencias y traumas de las personas al margen de la ley, necesidad de apoyo moral de la persona mayor, afectaciones y enfermedades crónicas e infecciosas, y reconocimiento de sus derechos humanos.

No se trata solo de priorizarlos por su edad mayor para obtener beneficios sociales y económicos, pues esta sería un forma de discriminación y de violentar su ejercicio de ciudadanía y su autonomía para participar en organizaciones sociales y políticos; sino de brindar un acompañamiento constante para garantizar que los procesos sean exitosos y generen transformaciones y mejoras reales a su calidad de vida, a la vez fortalecer las competencias de los profesionales que intervienen para responder a sus necesidades sentidas, desde el acompañamiento psicosocial en el componente específico, propuesto en la Resolución 1356 de 2016 (Agencia Colombiana para la Reintegración, 2016), modificatoria de la Resolución 754 de 2013 (Agencia Colombiana para la Reintegración, 2013).

Considerando que para una adecuada asistencia y reparación de daños derivados de la guerra, durante el proceso y después de su reintegración, es necesario la identificación de riesgos e impactos que ha dejado y continua dejando el tiempo de permanencia en los grupos al margen de la ley, entre los que se identifican: enfermedades y trastornos mentales por exposición al estrés constantey alejamiento de sus seres queridos; enfermedades físicas no diagnosticadas o tratadas debidamente; deterioro de la capacidad funcional por traumas y heridas; baja formación educativa puesto que muchos de ellos no alcanzaron niveles educativos altos (5 años en promedio de educación formal); ruptura de las redes sociales y menor número de personas que le brinden apoyo, ya que al estar fuera por espacios prolongados, las familias se adaptan a la ausencia de sus seres queridos y cuando ellos regresan, cada uno ya tiene construida su vida sin ellos; y el poco acceso a un empleo digno, pues su aprendizaje y experticia es en actividades ilegales.

La ARN en la dimensión salud de la ruta de reintegración (Agencia Colombiana para la Reintegración, 2017) se hace explícita la problemática mental derivada de la exposición a contextos de confrontación armada, lo que implica su abordaje desde lo individual, familiar y social, para mejorar su estado de salud y calidad de vida, favoreciendo el acceso al Sistema Ceneral de Seguridad Social en Salud (SCSSS) y a los programas del Ministerio de Salud y Protección Social (MSPS), superando la enfermedad y potencializando los recursos emocionales, cognitivos y mentales.

Para disminuir la sintomatología depresiva y aumentar el funcionamiento cognitivo de las PPRM, se sugiere la realización con apoyo del SCSSS de programas en línea de atención inmediata, de telesalud o de tecnologías de la información y la comunicación (TICS), con ello se detectarán oportunamente las alteraciones mentales que se presenten.

\section{Referencias bibliográficas}

Agencia Colombiana para la Reintegración [ACR]. (2017). Dimensiones de la Ruta de Reintegración. Conceptualización, logros, metas y categorías de avance. Bogotá: ACR.

Agencia Colombiana para la Reintegración. (24 de junio de 2016). Resolución número 1356 de 2016. Obtenido de https://www.ambitojuridico.com/BancoMedios/Documentos\%20PDF/res-1356-16(acr).pdf

Agencia Colombiana para la Reintegración. (25 de julio de 2013). Resolución 0754 de 2013. Obtenido de https://diario-oficial.vlex.com.co/vid/sticas-beneficios-desmovilizada-rdida-450907002

Amar ]., Llanos R., Ávila-Toscano J., Orozco C. (2011). Relación entre redes personales y calidad de vida en individuos desmovilizados del conflicto armado colombiano. Univ Psychol. 10(2), 355. 
Aristizábal E., Howe K., \& Palacio ]. (2009). Vulneración psicológica en víctimas y victimarios por efecto del conflicto armado en Magdalena, Atlántico, Cesar-Cuajira, Sucre y Bolivar. Revista de Psicología Universidad de Antioquia, 1(2), 7-25.

Baldovino M. (2014). Prevalencia de estrés postraumático y trastorno antisocial de la personalidad en la población desmovilizada de las guerrillas colombianas en Bogotá, D.C. [Tesis de maestría]. Bogotá: Universidad Católica de Colombia..

Belsky ]. (1990). The psychology of aging: Theory, research, and interventions. California: Brooks/Cole Pacific Grove.

Creamer M., Burgess P. \& McFarlane. A. (2001). Post-traumatic stress disorder: findings from the Australian National Survey of Mental Health and Well-being. Psychological Medicine 31 (7), 1237-1247.

Deeny P., \& McFetridge B. (2008). Critical care nursing in Northern Ireland: A rich history with a future of opportunity. Intensive Crit Care Nurs.24(3), 143-149.

Ebrahimzadeh M., Fattahi A. \& Nejad A. (2006). Long-term follow-up of Iranian veteran upper extremity amputees from the Iran-Iraq war (1980-1988). J Trauma Acute Care Surg. 61(4),886-888.

Fontana A., Rosenheck R. \& Desai R. (2010). Female veterans of Iraq and Afghanistan seeking care from VA specialized PTSD programs: Comparison with male veterans and female war zone veterans of previous eras. J Womens Health.19(4), 751-757.

Jiménez W. (2009). Salud mental en el posconflicto colombiano. Rev Crim. 51(1),179-192.

Korinek, K., \& Teerawichitchainan, B. (2014). Military service, exposure to trauma, and health in older adulthood: An analysis of northern vietnamese survivors of the Vietnam war. American Journal of Public Health, 104(8), 1478-1487.

Kulka R., Schlenger W., Fairbank J., Hough R., Jordan B., Marmar C., et al. (1990). Trauma and the Vietnam War Ceneration. New York: Brunner/Mazel.

Ministerio de Salud y Protección Social, Departamento Administrativo de Ciencia y Tecnología e Innovación, Colciencias, Universidad del Valle y Universidad de Caldas (2016a). Encuesta SABE Colombia: Situación de Salud, Bienestar y Envejecimiento en Colombia: Bogotá D.C., Colombia: PRECRAF IMPRESIONES S.A.S

Nakayama, R., Koyanagi, A., Stickley, A., Kondo, T., Gilmour, S., Arenliu, A., \& Shibuya, K. (2014). Social networks and mental health in post-conflict Mitrovica, Kosovo. BMC Public Health(14), 1169.

Organización Mundial de la Salud (2017a). La salud mental y los adultos mayores. Obtenido de https:// www.who.int/es/news-room/fact-sheets/detail/la-salud-mental-y-los-adultos-mayores

Organización Mundial de la Salud. (2017b). Salud mental y apoyo psicosocial en las emergencias. Obtenido de https://www.who.int/mental_health/emergencies/what_humanitarian_health_actors_should_know_spanish.pdf

Patel V., Araya R., Chatterjee S., Chisholm D., Cohen A., \& De Silva M., et al. (2007). Treatment and prevention of mental disorders in low-income and middle-income countries. The Lancet, 370(9591), 991-1005.

Peña L., Espíndola A., Cardoso ]., y Conzález T. (2007). La guerra como desastre. Sus consecuencias psicológicas. Humanidades Médicas, 7(3):1-7.

Pittman ]., Coldsmith A., Lemmer ]., Kilmer M. \& Baker D. (2012). Post-traumatic stress disorder, depression, and health-related quality of life in OEF/OIF veterans. Qual Life Res., 21(1), 99-103.

Preston, R. (1997). Integrating fighters after war: Reflections on the Namibian experience, 1989-1993. Journal of Southern African Studies, 23(3), 453-472.

Reeves R., Parker ]. \& Konkle-Parker D. (2016). War-related mental health problems of today's veterans: new clinical awareness. J Psychosoc Nurs Ment Health Serv., 43(7),18-28.

Richards A., Ospina-Duque ]., Barrera-Valencia M., Escobar-Rincón ]., Ardila-Cutiérrez M., Metzler T., et al. (2011). Posttraumatic stress disorder, anxiety and depression symptoms, and psychosocial treatment needs in Colombians internally displaced by armed conflict: A mixed-method evaluation. Psychol Trauma Theory Res Pract Policy., 3(4),384. 
Semana (enero 02 de 2017). La salud mental en Colombia, un enorme desafío del posconflicto [Internet]. Obtenido de http://www.semana.com/nacion/articulo/salud-mental-en-colombia-un-desafio-del-posconflicto/511266

Senarath U., Wickramage K., \& Peiris S. (2014). Prevalence of depression and its associated factors among patients attending primary care settings in the post-conflict Northern Province in Sri Lanka: a cross-sectional study. BMC Psychiatry, 14(1), 1.

Somasundaram, D., \& Sivayokan, S. (2013). Rebuilding community resilience in a post-war context: Developing insight and recommendations - a qualitative study in northern Sri Lanka. International Journal of Mental Health Systems, 7(3).

Stetz M., McDonald J., Lukey B., Gifford R. (2005). Psychiatric diagnoses as a cause of medical evacuation. Aviat Space Environ Med., 76(7), C15-C20.

Summerfield D. \& Toser L. (1991). "Low intensity”war and mental trauma in Nicaragua: A study in a rural community. Med War., 7(2), 84-99.

Wilmer, F. (1998). The social construction of conflict and reconciliation in the former Yugoslavia. Social Justice, 25(4), 90-113.

World Health Organization. (2004). Prevention of mental disorders. Effective interventions and policy options. Geneva: WHO.

Nota.

'Esta publicación se realiza en el marco del proyecto "Fortalecimiento para la prestación de servicios a excombatientes mayores de 50 años, en proceso de reintegración con la Agencia para la Reincorporación y la Normalización (ARN), anteriormente Agencia Colombiana de Reintegración (ACR)". El proyecto fue realizado por FUNDACOL y la ARN y la Organización Internacional para las Migraciones (OIM), con el apoyo de la Agencia de Estados Unidos para el Desarrollo Internacional (USAID). Los contenidos son responsabilidad de los autores y no necesariamente reflejan las opiniones de la USAID o el gobierno de los Estados Unidos de América, ni de la OIM. 